INTERNATIONAL JOURNAL OF SYSTEMATIC BACTERIOLOGY

Vol. 17, No. 3 July 1967

pp. $269-272$

Copyright 1967, Iowa State University Press

\title{
DECAR BOXYLATION OF L-HISTIDINE BY CLOSTRIDIA ${ }^{\prime}$
}

Marilyn Friedrich, Sheldon Steiner and Ralph H. Weaver

\author{
Department of Microbiology \\ University of Kentucky, Lexington, Kentucky
}

SUMMARY. A porcelain plate microtest by which the decarboxylation of L-histidine by clostridia could be detected within 6 hours is described. It was more sensitive than chromatographic procedures for detecting histamine production, using ninhydrin or Pauly's reagent as a developing agent, which were used for confirmation. All of 26 type A strains of Clostridium perfringens and the one type F strain tested decarboxylated histidine. Strongly positive tests were obtained except for some of the Hobbs type strains that gave much weaker reactions. Two type C strains gave negative tests. Fifteen of 19 strains of Clostridium bifermentans also produced decarboxylation, but the reactions were weak and inconsistent. No strains of 18 other species of clostridia tested produced decarboxylation.

Gale $(1941,1946)$ reported that 9 of 10 strains of Clostridium perfringens, type $A$, as well as strains of types $B, C$, and $D$, decarboxylated histidine; it was also reported that

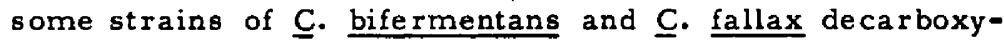
lated it more slowly. He detected decarboxylase production by the evolution of carbon dioxide by washed-cell suspensions of organisms in a manometer.

We have tested a more extensive series of strains of clostridia to determine the taxonomic significance of histidine-decarboxylase production and have investigated simpli-

1 This work was supported by NSF Undergraduate Research Participation grant GE-4148 and Public Health Service Research Grant AI 04958 from the National Institute of Allergy and Infectious Diseases. 
fied procedures for its detection in an attempt to facilitate routine systematic work.

Modification of the tests of Moeller (1955) and Falkow (1958) that were useful to detect other amino-acid decarboxylases by clostridia could not be used with histidine because of its strong buffering action. The Shaw and Clark (1955) test to detect decarboxylation of histidine by members of the proteus-providence group gave negative results with clostridia.

A rapid micromethod used by Steiner (unpublished) for the study of other decarboxylases of clostridia was modified for use with histidine, and the results were confirmed by chromatography.

\section{MATERLALS AND METHODS}

\section{Micromethod}

Substrate: Histidine solutions of $0.006 \mathrm{M}$ and $0.003 \mathrm{M}$ concentration gave equally good results. Higher concentrations were unsatisfactory because of buffering action, and lower ones gave weak reactions. One to $2 \mathrm{mg}$ amounts of pyridoxal phosphate and ferric chloride per $100 \mathrm{ml}$ of solution we re added, and the solutions were adjusted to $\mathrm{pH} 3.8$ or 3.2. When the solutions were adjusted to $\mathrm{pH} 3.8,4 \mathrm{ml}$ of a saturated aqueous solution of bromcresol green were added and when they were adjusted to $\mathrm{pH} 3.2,4 \mathrm{ml}$ of a $0.004 \%$ solution of methyl redwere used. Equivalent results were obtained at the two $\mathrm{pH}$ values. The substrate solutions can be stored in the refrigerator if a drop of chloroform and one of toluene are added.

Cell suspensions: Cultures were grown for 24 hours at $35^{\circ} \mathrm{C}$ in fluid thioglycollate medium and were used for inoculation with capillary pipettes into screw-cap tubes filled with $16 \mathrm{ml}$ amounts of medium containing casitone, $15 \%$; glucose, $2 \%$; yeast extract, $3 \%$; sodium thioglycollate, $0.5 \%$; $\mathrm{NaCl}, 2.5 \%$; and L-histidine, $0.5 \%$. After incubation for 24 hours, the cells were concentrated by centrifugation, washed once with acidified distilled water at $\mathrm{pH} 4.0$, and resuspended in $0.5-\mathrm{ml}$ amounts of the acidified water.

Microtest: One to 2 drop amounts of cell suspension were added to 4 to 6 drop amounts of amino-acid substrate solution in depressions in Coors white porcelain color reaction plates. The plates were then covered with Saran Wrap and incubated at $35^{\circ} \mathrm{C}$. Results were read after 6 and 24 hours, a positive test consisting of a shift from yellow to blue with 
bromcresol green and from red to yellow with methyl red. Apparently the $6-\mathrm{hr}$ reading can be considered final since no further positives were found after 24 hours.

\section{Chromatographic procedure}

Cell suspensions and L-histidine substrate solutions were incubated in depressions in the porcelain plates as in the micromethod and were tested for histamine. However, it was necessary to use a $0.05 \mathrm{M}(1 \%)$ solution of histidine, adjusted to $\mathrm{pH} 3.8$, and incubate for 24 hours. The higher concentration of histidine and longer incubation were necessary because the chromatographic procedure is less sensitive than the microtest. Negative results were obtained when the tests were run at $\mathrm{pH} 2.5$ to 3.0 , the $\mathrm{pH}$ used by Gale in his manometric tests.

For chromatography, the reaction mixtures were spotted on Whatman No. 4 paper with capillary tubes, a central spot of histidine solution and one of a histidine-histamine mixture being placed on each chromatogram. The solvent used was $77 \%$ ethanol. Duplicate chromatograms were made. One was dipped in $0.2 \%$ ninhydrin in acetone while the other was sprayed with Pauly's reagent after Kutacek (Hais and Macek 1963). With the ninhydrin the histamine spot appears purple while with Pauly's reagent it is a deep cherry red on a yellow background. While Pauly's reagent, which reacts with imidazoles, is more specific than ninhydrin, which reacts with primary amines, it is less sensitive.

Cultures: All strains used were obtained from reliable sources. They were tested for purity and, insofar as possible, for correctness of species designation.

\section{RESULTS}

Clostridium perfringens strains bearing NCTC designations representing Hobbs types $1,3,5,7$ and 12 gave weak and inconsistent tests for histidine decarboxylase production by the microtest procedure and weak or negative results with the chromatographic procedure. The type 8 strain was a stronger histidine-decarboxylase producer. The Hobbs type 9 strain and 19 other type A strains gave consistently positive results with the microtest, which were confirmed chromatographically, as did the one type $F$ strain. Two type $C$ strains failed to produce demonstrable histidinedecarboxylase activity. In view of the report of production of this enzyme by type C strains by Gale (1946), this result 
may represent variation rather than a characteristic of type C strains.

Of 19 strains of Clostridium bifermentans tested, 4 failed to demonstrate histidine-decarboxylase activity and 15 gave weak and inconsistent tests with the microprocedure; 7 could be confirmed chromatographically.

The following species were found not to produce histidine decarboxylase (the numbers in parentheses indicate the

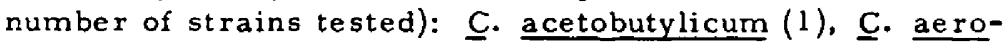
foetidum (2), C. beijerinckii (1), C. botulinum (19, including types $A, B, C$ and $E$ ), $\underline{C}$. capitovale (2), $\underline{C}$. carnis (1), C. chauvoei ( 1 ), C. cochlearium (1), C. difficile (2), C. histolyticum (1), C. lentoputrescens (1), C. novyi (3), ‥ para-

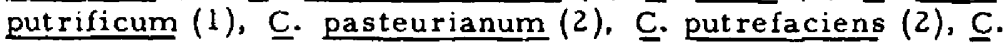
septicum (3), C. sporogenes (7), and $\underline{C}$. tetani (11). One culture labelled $\underline{C}$. sporogenes gave positive tests but was subsequently found to be a mixed culture, one of the isolates having the characteristics of $\mathrm{C}$. bifermentans. A culture labelled $\underline{C}$. histolyticum also gave positive tests; it appears to be a pure culture but does not have the characteristics of C. histolyticum. Its identity is uncertain.

The results of this study appear to be in good agreement with those of Gale $(1941,1946)$. C. perfringens is a strong histidine-decarboxylase produce $r$ and $\underline{C}$. bifermentans is a weak producer. Gale reported positive tests with one strain

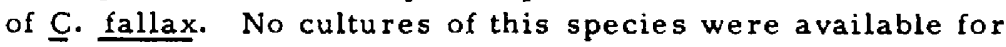
our study.

\section{REFERENCES}

Clark, P. H. and C. Shaw. 1955. Biochemical classification of proteus and providence cultures. J. gen. Microbiol. $13: 155-161$.

Falkow, S. 1958. Activity of lysine decarboxylase as an aid in the identification of Salmonellae and Shigellae. Am. J. Clin. Pathol. 29:598-600.

Gale, E.F. 1941. The decarboxylation of amino-acids by organisms of the groups clostridium and proteus.

Biochem. J. 35:66-79.

- 1946. The bacterial amino acid decarboxylases. Advances in Enzymology 6:1-32.

Hais, I. M. and K. Macek. 1963. Paper Chromatography. Academic Press, New York: p. 786.

Moeller, V. 1955. Simplified tests for some amino acid decarboxylases and for the arginine dihydrolase system. Acta Path. Microbiol. Scand. 36:158-172. 